over half of teens 15-19 years reported having had oral sex with an opposite sex partner. Oral sex is thought by some men who have sex with men to have lower risk for HIV acquisition, and is a prevalent practice in this population. However, the risk of acquiring a given sexually transmitted infection (STI) from oral sex may depend on a number of factors, including the specific STI, particular oral sex practice, prevalence of the STI in the population, and the number of oral sex acts performed. It is difficult to quantify the risk of getting an STI from a specific type of oral sex activity, or compare risks due to specific sex acts, because most people who have oral sex also have vaginal and/or anal sex.

Methods In order to develop public health messages on oral sex and STI risk, we performed a review of the scientific literature to assess the state of current knowledge for risks of seven STIs (including HIV) and six oral sex practices: insertive and receptive fellatio, cunnilingus, and anilingus. Approximately 200 publications between 1979 and 2010 were reviewed; only 65 reported novel findings for one or more of these 42 potential associations.

Results The clearest evidence of risk was found for syphilis (all oral sex practices), chlamydia (insertive and receptive fellatio; receptive anilingus), gonorrhoea (insertive and receptive fellatio), herpes (insertive fellatio; receptive cunnilingus), and HIV (receptive fellatio). For example, for pharyngeal gonorrhoea 4 of 4 studies reported association with receptive fellatio; 1 of 1 study found none for insertive anilingus. Other STI and oral sex associations were less well-studied, or have not been documented. No reports allow direct per-contact estimates, but some facilitate comparisons with non-oral types of sexual contact. A number of studies did not specify whether ejaculation or use of barrier protection was assessed.

Conclusions All oral sex practices are associated with risk of transmission of one or more STIs; however the relations between specific oral sex practices and risk of infection are not well-characterised for several STIs. Despite this, it is important to formulate appropriate public health messages regarding oral sex and STI risk. Future studies should clearly distinguish between specific oral sex practices and consider these factors in sample size determination.

\section{P1-S5.07 ONGOING RISK BEHAVIOURS: NEW STD DIAGNOSES IN PERSONS KNOWN TO BE HIV-INFECTED IN MIAMI-FORT LAUDERDALE (SOUTH FLORIDA), 2000-2009}

doi:10.1136/sextrans-2011-050108.185

${ }^{1} \mathrm{~T}$ A Peterman, ${ }^{2} \mathrm{~T}$ H Brewer, ${ }^{1} \mathrm{D}$ R Newman, ${ }^{3} \mathrm{~S}$ Shiver. ${ }^{1} \mathrm{CDC}$, Atlanta, USA; ${ }^{2} \mathrm{CDC} /$ Florida DOH, Miami, USA; ${ }^{3}$ Florida Department of Health, Tallahassee, USA

Background The diagnosis of a new bacterial STD in a person with documented HIV- infection suggests ongoing high-risk sexual behaviours. While high rates of syphilis among HIV-positive MSM have been repeatedly noted in South Florida, little attention has been given to the incidence of gonorrhoea (GC) and chlamydia (CT) among HIV-positive individuals, due to use of separate surveillance systems, differing security requirements, and limited partner services staff available to investigate GC and CT cases.

Methods We reviewed STD surveillance data from 2000 to 2009 after linking it to the HIV/AIDS surveillance data base. We analysed cases of GC and CT diagnosed $>60$ days after an HIV diagnosis.

Results Overall $3.7 \%$ of GC (1504 of 40214 ) and $1.2 \%$ of CT (1149/ 99265) cases were in persons known to be HIV- positive. The percentage of cases diagnosed in HIV- positive persons increased steadily over the time period; GC coinfections increased from $2.3 \%$ in 2000 to $5.0 \%$ in 2009 and CT coinfections increased from $0.8 \%$ to $1.3 \%$. Most of the GC coinfected cases were among males; 1144/
$21360(5.4 \%)$ of males with GC were coinfected with HIV while $360 / 18854(1.9 \%)$ of females with GC were coinfected with HIV. For CT, $559 / 22735(2.5 \%)$ of males with CT were coinfected with HIV while $590 / 76530$ (0.8\%) of females with CT were coinfected with HIV. Most coinfections were among HIV positive males, 73.5\% of whom were men who have sex with men (MSM). Coinfections among men increased over the time period, while among women the number peaked in 2006 and has declined thereafter. HIV-positive men accounted for over $10 \%$ of GC infections among men $35-59$ years of age and over $10 \%$ of CT infections among males 40-59. HIV coinfection was present for over $5 \%$ of GC infections among women $30-44$ years of age and $50-54$ years of age.

Conclusions While males with GC or CT who are coinfected with HIV constitute a small fraction of the cases in South Florida, the proportion is increasing steadily. HIV+ MSM account for a high percentage of cases among males over 35 years of age. Timely, accessible linkage between HIV and STD databases is essential to direct partner services and risk reduction counselling to this high-risk population.

\section{P1-S5.08 ATTENDANCE AT LOCAL AND NATIONAL SEX-EVENTS ASSOCIATED WITH STD DIAGNOSIS, SAN FRANCISCO, 2010}

doi:10.1136/sextrans-2011-050108.186

K Bernstein, H Hjord, J Marcus, R Kohn, S Philip. San Francisco Department of Public Health, San Francisco, USA

Background A variety of sex-themed events draw crowds from across the USA and provide opportunities for sex partner recruitment, potentially amplifying local sexual networks and complicating disease prevention and control. We examined the frequency of attendance at such events and the association between attendance and incident STD diagnosis among patients visiting a municipal STD clinic.

Methods During 21 September 10-1 November 10, patients seeking services at San Francisco City Clinic were invited to complete a survey about their attendance at local and national events in the prior 12 months, including street fairs, gay pride events, Burning Man, leather events, and circuit parties. Survey results were linked with data from the clinic visit, including self-reported risk behaviours and STD diagnoses. Analyses were stratified by sexual orientation. Events traditionally associated with sex partner recruitment (sex events) were combined. $\chi^{2}$ and Wilcoxon rank-sum statistics were used to compare patients who attended sex events with all other patients completing the survey.

Results Of the 246 completed surveys, 106 (43.1\%) were among heterosexual patients and 140 (56.9\%) were among men who have sex with men (MSM). Twenty per cent of heterosexual patients and $47 \%$ of MSM patients reported attending a sex event in the prior 12 months. Among heterosexual patients, those attending a sex event were of younger age (median 25 vs 29 years, $p=0.014)$, reported fewer sex partners in the prior 12 months (median 1 vs $2, p=0.021$ ) and were more likely to be at their first visit to the STD clinic $(71.4 \%$ vs $47.1 \%, 0.045)$. There was no association between STD diagnosis and event attendance among heterosexuals. Among MSM, those attending sex events reported more sex partners in the prior 12 months (median $10 \mathrm{vs} 5, \mathrm{p}=0.019$ ), were more likely to be residents of San Francisco (83.3\% vs $66.2 \%$, $\mathrm{p}=0.021$ ), and were more likely to be diagnosed with chlamydia ( $15.2 \%$ vs $4.1 \%, p=0.024)$ or any STD $(29.0 \%$ vs $14.9 \%, p=0.045)$ at that visit.

Conclusions Attendance at sex events was common among patients at an urban STD clinic. Among MSM, attendance was associated with more sex partners and STD diagnosis. The ability of local 
programs to successfully prevent and control STDs may be complicated by recruitment of new sex partners at such events. Innovative collaborative prevention interventions at sex events and for residents returning from such events should be explored.

\section{P1-S5.09 ABSTRACT WITHDRAWN}

\section{P1-S5.10 ACUTE GENITAL ULCERATION IN SOUTH AFRICAN MEN: IMPORTANCE OF AGE AND SEXUAL RISK BEHAVIOURS}

\author{
doi:10.1136/sextrans-2011-050108.188
}

${ }^{1} \mathrm{~J}$ Leichliter, ${ }^{2} \mathrm{D}$ Lewis, ${ }^{3} \mathrm{G}$ Paz-Bailey. ${ }^{1}$ Centers for Disease Control \& Prevention, Atlanta, USA; ${ }^{2}$ NICD/NHLS, South Africa; ${ }^{3}$ Universidad del Valle de Guatemala, Guatemala

Background Herpes simplex virus (HSV) is the leading cause of genital ulcer disease (GUD) in Africa. Although some studies have examined the demographics and sexual behaviours of men with GUD, less is known about the differences that may exist between men with new sexually-acquired ulcers and men with other forms of GUD.

Methods Men with GUD ( $\mathrm{n}=615)$, who were 18 years and older, were recruited from several primary healthcare clinics in Gauteng Province, South Africa. Participants received syndromic management, were tested for ulcer aetiology and urethritis pathogens by PCR, and for HIV, syphilis and HSV-2 by serology. We used survey and STI test data to examine correlates of initial vs recurrent HSV and acute ulcer outcomes. Of men with HSV $(n=451)$, initial HSV was defined as a HSV-positive ulcer specimen and HSV-negative serology. Recurrent herpes was defined as a positive HSV specimen and serology. For all men, the acute ulcer outcome compared men who had initial HSV or who had Treponema pallidum, Haemophilus ducreyi, or Chlamydia trachomatis L1-L3 detected in their ulcer specimens (termed "acute ulcers") to men with GUD who did not have any of the four etiologies (termed "non-acute ulcers"). Correlates consisted of demographic characteristics and sexual behaviours often associated with STI.

Results Among all men, 29\% had an acute ulcer, and of those with HSV, 30\% had initial HSV. As compared to men with a non-acute ulcer, men with an acute ulcer were younger $(p<0.01)$, more likely to be single $(p<0.05)$, less likely to always use condoms with regular partners $(p=0.03)$, had more casual partners in the last 3 months $(p=0.03)$ but had fewer lifetime partners $(p=0.02)$. As compared to men with recurrent HSV, men with initial HSV were significantly more likely to be younger $(p<0.01)$, have multiple regular partners $(p=0.03)$, and report having sex in last week $(p=0.04)$, but were less likely to report ever exchanging sex for money $(p=0.01)$ and had fewer lifetime partners $(p<0.01)$.
Conclusions Findings suggest that young age and recent sexual behaviour were associated with initial HSV and acute ulcers in this group of men. These data emphasise the importance of targeting STI/HIV prevention programs in South Africa to young men engaging in high-risk sexual activities. To facilitate this, it is important to ensure that young men are equipped with the appropriate knowledge and skills to either avoid acquiring, or to seek early treatment for, STIs.

\section{P1-S5.11 HIGH PREVALENCE OF STIS AND RISK BEHAVIOURS AMONG PERSONS LIVING WITH HIV IN NICARAGUA: MISSED OPPORTUNITIES FOR PREVENTION}

doi:10.1136/sextrans-2011-050108.189

${ }^{1} \mathrm{~J}$ Goins, ${ }^{1} \mathrm{~L}$ M Romero, ${ }^{1} \mathrm{~F}$ de María Hernandez, ${ }^{1} \mathrm{~S}$ Delgado, ${ }^{1} \mathrm{~B}$ Alvarez, ${ }^{2} \mathrm{E}$ Beteta, ${ }^{3} \mathrm{G}$ Paz-Bailey, ${ }^{1} \mathrm{~S}$ Morales. 'Universidad del Valle, Guatemala City, Guatemala; ${ }^{2}$ Ministerio de Salud de Nicaragua, Managua, Nicaragua; ${ }^{3}$ Tephinet, Atlanta, USA

Background The UNAIDS Report on the Global AIDS Epidemic estimated that in 2009 Nicaragua had 6900 cases of HIV, almost double the 2001 estimate. Among people living with HIV in Nicaragua, it remains important to monitor sexually transmitted infections (STIs) and behavioural risk factors in order to inform STI prevention programs about the health needs of this population.

Methods In this cross sectional study, 200 people living with HIV were recruited consecutively from patients attending the Roberto Calderon Hospital in Managua, Nicaragua. Data was collected using computer-assisted self-interviews. Both men and women were tested for active syphilis (RPR titer $\geq 1: 8$ ) and herpes simplex virus type 2 (HSV-2) by serology, and for Chlamydia trachomatis, Neisseria gonorrhoeae, Trichomonas vaginalis, and Mycoplasma genitalium, by PCR. Acute STI was defined as infection with active syphilis or any of the PCR diagnosed STIs. We examined associations between acute STI infection and reported condom use as well as between STI infection and gender using simple logistic regression.

Results The most prevalent STI among study participants was HSV-2 (81.5\%). Active syphilis was diagnosed in $6.0 \%$ of the population. Men were less likely to be infected with an acute STI than women $(\mathrm{OR}=0.24 ; 95 \%$ CI 0.10 to 0.57 , Abstract P1-S5.11 table 1). A high percentage of participants reported having an HIV positive stable partner at the time of the interview (85.1\%). Consistent condom use in the last year was reported to be $59.9 \%$ with stable partners, $66.7 \%$ with commercial sex partners and $74.6 \%$ with occasional partners. Condom use at last sex was found to be protective for infection with acute STI $(\mathrm{OR}=0.38$; $95 \% \mathrm{CI} 0.17$ to 0.85).

Abstract P1-S5.11 Table 1 Prevalence of STls among persons living with HIV and OR by gender

\begin{tabular}{|c|c|c|c|c|c|c|c|c|c|c|}
\hline & \multicolumn{3}{|c|}{ Total } & \multicolumn{3}{|l|}{ Men } & \multicolumn{3}{|c|}{ Women } & \multirow[b]{2}{*}{$O R^{*}(95 \% \mathrm{CI})$} \\
\hline & $\mathbf{N}$ & $\mathbf{n}$ & $\%(95 \% \mathrm{Cl})$ & $\mathbf{N}$ & $\mathbf{n}$ & $\%(95 \% \mathrm{CI})$ & $\mathbf{N}$ & n & $\%(95 \% \mathrm{CI})$ & \\
\hline HSV-2 & 200 & 163 & 81.5 (75.4 to 86.6$)$ & 107 & 84 & 78.5 (69.5 to 85.9$)$ & 93 & 79 & 85.0 (76.0 to 91.5$)$ & $0.65(0.31$ to 1.35$)$ \\
\hline Syphilis & 199 & 23 & $11.6(7.5$ to 16.8$)$ & 106 & 13 & $12.3(6.7$ to 20.1$)$ & 93 & 10 & 10.8 (5.3 to 18.9$)$ & $1.16(0.48$ to 2.79$)$ \\
\hline Active syphilis & 199 & 12 & $6.0(3.2$ to 10.3$)$ & 106 & 6 & 5.7 (2.1 to 11.9$)$ & 93 & 6 & $6.5(2.4$ to 13.5$)$ & 0.87 (0.27 to 2.80$)$ \\
\hline Bacterial vaginosis & 81 & 23 & $28.4(18.9$ to 39.5$)$ & - & - & - & 81 & 23 & $28.4(18.9$ to 39.5$)$ & - \\
\hline Chlamydia trachomatis & 198 & 4 & $2.0(0.6$ to 5.1$)$ & 107 & 1 & $0.9(0.0$ to 5.1$)$ & 91 & 3 & $3.3(0.7$ to 9.3$)$ & $0.28(0.03$ to 2.71$)$ \\
\hline Neisseria gonorrhoeae & 198 & 0 & $0(0.0$ to 1.8$) \dagger$ & 107 & 0 & $0(0.0$ to 3.4$) \dagger$ & 91 & 0 & $0(0.0$ to 4.0$) \dagger$ & - \\
\hline Mycoplasma gentitalium & 198 & 9 & $4.6(2.1$ to 8.5$)$ & 107 & 1 & $0.9(0.0$ to 5.1$)$ & 91 & 8 & 8.8 (3.9 to 16.6$)$ & $0.10 \neq(0.01$ to 0.80$)$ \\
\hline Trichomonas vaginalis & 198 & 12 & 6.1 (3.2 to 10.6$)$ & 107 & 0 & $0(0.0$ to 3.4$) \dagger$ & 91 & 12 & 13.19 (7.0 to 21.9$)$ & - \\
\hline Any acute STI & 197 & 31 & 15.7 (10.9 to 21.6$)$ & 106 & 8 & $7.5(3.3$ to 14.3$)$ & 91 & 23 & 25.3 (16.7 to 35.5$)$ & $0.24 \S(0.10$ to 0.57$)$ \\
\hline
\end{tabular}

${ }^{*}$ Referent gender category: Women

†One-sided, $97.5 \% \mathrm{Cl}$.

$\neq \mathrm{p}$ value $<0.05$.

sp value $=0.001$

\Active syphilis, Chlamydia trachomatis, Neisseria gonorrhoeae, Mycoplasma gentitalium or Trichomonas vaginalis. 